DFTUZ 95-01

HUPD 9505

January, 1995

\title{
One loop renormalization of the four-dimensional theory for quantum dilaton gravity
}

\author{
ILYA L. SHAPIRO \\ Departamento de Fisica Teorica, Universidad de Zaragoza, 50009, Zaragoza, Spain. \& \\ HiROYUKI TAKATA \\ Department of Physics, Hiroshima University, Higashi-Hiroshima 724, Japan. [
}

\begin{abstract}
We study the one loop renormalization in the most general metric-dilaton theory with the second derivative terms only. Classical action includes three arbitrary functions of dilaton. The general theory can be divided into two classes, models of one are equivalent to conformally coupled with gravity scalar field and also to general relativity with cosmological term. The models of second class have one extra degree of freedom which corresponds to dilaton. We calculate the one loop divergences for the models of second class and find that the theory is not renormalizable off mass shell. At the same time the arbitrary functions of dilaton in the starting action can be fine-tuned in such a manner that all the higher derivative counterterms disappear on shell. The only structures in both classical action and counterterms, which survive on shell, are the potential (cosmological) ones. They can be removed by renormalization of the dilaton field which acquire the nontrivial anomalous dimension, that leads to the effective running of the cosmological constant. For some of the renormalizable solutions of the theory the observable low energy value of the cosmological constant is small as compared with the Newtonian constant. We also discuss another application of our result. In particular, our calculations in a general dilaton model in original variables give the possibility to estimate quantum effects in $\Lambda+\alpha R+\beta R^{2}$ theory.
\end{abstract}

\footnotetext{
${ }^{1}$ On leave from Tomsk Pedagogical Institute, 634041 Tomsk, Russia. E-mail: shapiro@dftuz.unizar.es

${ }^{2}$ E-mail: takata@theo.phys.sci.hiroshima-u.ac.jp
} 


\section{Introduction}

Recently it has been a considerable interest to the metric - scalar gravity in four dimensions. The active research in this field was inspired by different reasons (see [1] for the interesting discussion of the subject). In particular, the effective action of (super)string depends on both metric and dilaton (see, for example, [2]). Such an effective action arise in a form of the power series in a string loop parameter $\alpha^{\prime}$, and the standard point of view is that the higher orders in such an expansion correspond to higher energies. From this point of view at lower energy scale the action for gravity has the form of the lower derivative dilaton action. From another hand the presence of dilaton in a low derivative gravity action leads to the inflationary cosmological solution, that enables one to solve some specific problems in the field of cosmology. The problem of classical solutions and the cosmological phase transitions in a dilaton theory has been extensively studied (see, for example, [3, 4, 5, 6, 7]). Moreover it turned out that some special version of the dilaton gravity is classically equivalent to the restricted higher derivative gravity theory which include only square of scalar curvature in addition to Hilbert-Einstein action [8, 3, 9] (see also the last paper for more complete references). This theory is also of big cosmological interest because it enables one to construct the inflationary solutions [10, 11, 12, 13, 6]).

Perhaps the completely consistent theory of quantum gravity can be constructed within the string model, and gravity will be described by effective action within this frame. However the string theory can be valid at the Planck energies and above, and if one wish to deal with the energies below Planck scale, it is natural to suppose that the quantum effects of gravity will be related with some low energy action. One can, for instance, apply higher derivative gravity for this purposes. Higher derivative gravity is renormalizable [14, 15] and allow the renormalization group study of some physical effects like asymptotic freedom [16, 17, 18] and phase transitions [28, 29], but not unitary (at least within the usual perturbation scheme (see 222 for the introduction and more complete references). Thus at the moment we do not have any consistent theory which is applicable below Planck scale and any researchment in this field is based on the choice of some model, which allow us to explore some quantum gravity effects. In present paper we consider the four dimensional metric-dilaton model including the second derivative terms only. We choose the most general action including arbitrary functions $A, B, C$ of the dilaton $\phi$.

$$
S=\int d^{4} x \sqrt{-g}\left\{A(\phi) g^{\mu \nu} \partial_{\mu} \phi \partial_{\nu} \phi+B(\phi) R+C(\phi)\right\}
$$

that covers all special cases including the string inspired action, special (relevant from cosmological viewpoint) case of higher derivative gravity and also admit some other interesting applications. Such a model is non-renormalizable that can be seen already from power counting consideration. Indeed one can suppose that all necessary counterterms are introduced from the very beginning, but then the finite parts of the amplitudes and also the "beta functions" for the generalized couplings will be ill defined because of relevant gauge fixing and parametrization dependence and therefore any analysis becomes inconsistent. However there are a few possibilities to obtain some sound results for the theory (1) on quantum level. First of all there is some interest to explore the one-loop renormalization of the theory and to compare the results with the ones for General Relativity [23]. In the last case all the one-loop counterterms vanish (if the cosmological term is lacking) on mass shell and hence the one-loop $S$-matrix is finite. The theory with cosmological constant is renormalizable 
[20], however if one introduce the matter fields the one loop renormalizability is lacking even on mass shell. It should be interesting to know, whether it is so for the dilaton model (1). In this case the situation is much more complicated, because the amount of possible counterterms is essentially higher as compared with the pure metric theory. It turns out, however, that it is possible to reduce the counterterms to the few structures which survive on mass shell.

Furthermore, if the consideration is restricted by the one-loop on shell case, then the theory with cosmological term $C(\phi)$ can be renormalizable that leads to some general conjectures about the high energy behavior of quantum gravity [16]. Next, we can restrict ourselves by some special backgrounds where the theory is renormalizable. For example, the cosmological inflationary background provides the renormalizability of the special higher derivative model which is the particular case of the above model [24]. On the other hand one can introduce an additional constraint on the background dilaton and regard it as constant. This way is also of some cosmological interest, because the renormalizability in the potential sector enables one to evaluate the significance of quantum gravity for the cosmological phase transitions.

The action (1) may be viewed as the second derivative part of the general (fourth derivative) model of the dilaton gravity, which has been recently inversigated in [25]. In [25] we have restricted ourselves by the case when only the scalar field is the quantum variable. Despite the general case is very interesting, the explicit calculations are too cumbersome because of the presence of higher derivatives. Here we perform the one-loop calculations in the theory (11), considering both fields $\phi$ and $g_{\mu \nu}$ as quantum ones. We start with the general model (11) and then turn to the analysis of special cases.

The paper is organized as follows. In section 2 we discuss the different conformally equivalent forms of the theory (四), and show that all of them can be divided into two sets. Models of one set are classically equivalent to conformal scalar - metric theory and, simultaneously, to General Relativity. The models of second set include physical degrees of freedom, corresponding to the dilaton (or conformal factor), and in forthcoming sections we restrict the consideration only by the models of this class. In section 3 the general structure of renormalization of (1) is explored both off and on mass shell. In section 4 we calculate of the one-loop counter terms. To make this we apply the method which was developed in 27] within the two dimensional dilaton gravity. It turns out that it is useful in $d=4$ as well, and not only in the model (1) but also in the higher derivative dilaton gravity formulated recently in 25] (see also discussion in [19]). In section 5 the concrete analysis of the on shell renormalization of the model is performed. Here we fine tune the functions $A$ and $B$ to provide one-loop finiteness of the theory without $C$ term. If the potential term is included then the one loop on shell renormalizability require the vanishing of the Einstein counterterm. As a result we face with the cosmological type divergences only, and it turns out that they can be removed by renormalization of the scalar dilaton field. In section 6 the renormalization of the dilaton theory (1) interacted with matter fields is discussed. It turns out that qualitatively the structure of counterterms is the same as in the Einstein gravity, and the dilaton - metric theory with matter is non-renormalizable even on mass shell. In section 7 we give the qualitative discussion of the renormalization in two special cases, one of them is rather interesting and has to be analyzed separately. The last section consists in discussion of the results. 


\section{General notes on the dilaton gravity}

If we are interested to understand the parametrization dependence of the dilaton action, it is useful to start with the simple particular case of the general action (11).

$$
S=\int d^{4} x \sqrt{-g^{\prime}}\left\{R^{\prime} \Phi+V(\Phi)\right\}
$$

Here the curvature $R^{\prime}$ corresponds to the metric $g_{\mu \nu}^{\prime}$ and $g^{\prime}=\operatorname{det}\left(g_{\mu \nu}^{\prime}\right)$. Let now transform this action to new variables $g_{\mu \nu}$ and $\phi$ according to

$$
g_{\mu \nu}^{\prime}=g_{\mu \nu} e^{2 \sigma(\phi)}, \quad \Phi=\Phi(\phi)
$$

where $\sigma(\phi)$ and $\Phi(\phi)$ are arbitrary functions of $\phi$. In a new variables the action becomes:

$$
S=\int d^{4} x \sqrt{-g}\left\{\Phi(\phi) R e^{2 \sigma(\phi)}+6(\nabla \phi)^{2} e^{2 \sigma(\phi)}\left[\Phi \sigma^{\prime}+\Phi^{\prime}\right] \sigma^{\prime}+V(\Phi(\phi)) e^{4 \sigma(\phi)}\right\}
$$

Therefore we are able to transform the particular action (2) to the general form (1) with

$$
A(\phi)=6 e^{2 \sigma(\phi)}\left[\Phi \sigma^{\prime}+\Phi^{\prime}\right] \sigma^{\prime}, \quad B(\phi)=\Phi(\phi) e^{2 \sigma}
$$

It is quite reasonable to explore the inverse problem, that is to find the form of $\sigma(\phi)$ and $\Phi(\phi)$ that correspond to the given $A(\phi)$ and $B(\phi)$. One can find that in this case $\sigma(\phi)$ and $\Phi(\phi)$ are defined from the equations

$$
A=6 B_{1} \sigma_{1}-6 B\left(\sigma_{1}\right)^{2}, \quad \Phi=B e^{-2 \sigma}
$$

Substituting (6) into (4) we find that in a new variables the action have the form

$$
S=\int d^{4} x \sqrt{-g}\left\{A(\phi) g^{\mu \nu} \partial_{\mu} \phi \partial_{\nu} \phi+B(\phi) R+\left(\frac{B}{\Phi}\right)^{2} V(\Phi(\phi))\right\}
$$

where the last term is nothing but $C(\phi)$ from(11).

It is easy to see that the above transformations lead to some restrictions on the functions $A(\phi)$ and $B(\phi)$. Let us consider the special case of Einstein gravity that is to put $\Phi=$ const. One can rewrite this condition in terms of $A(\phi)$ and $B(\phi)$. Note that

$$
2 A B-3\left(B_{1}\right)^{2}=-3\left(\frac{d \Phi}{d \phi}\right)^{2} e^{4 \sigma(\phi)}
$$

Here and below the lower index show the order of derivative with respect to $\phi$. For instance,

$$
B_{1}=\frac{d B}{d \phi}, \quad A_{2}=\frac{d^{2} B}{d \phi^{2}}, \quad \sigma_{1}=\frac{d \sigma}{d \phi}, \quad \text { etc. }
$$

Hence it is clear that the case of $2 A B-3\left(B_{1}\right)^{2}=0$ qualitatively differs from another ones. Let us now comment this amusing case. We start with the most simple example $A=1, B=\xi \phi^{2}$ where $\xi=\frac{1}{6}$. Then the equation (6) can be easily solved and we obtain $\sigma(\phi)=\sigma_{0}+\ln |\phi|$ and $\Phi=\frac{1}{6} e^{2 \sigma_{0}}=$ const. Next, substituting these expressions into (团) we find that in a new variables the transformed action has the form

$$
S=\int d^{4} x \sqrt{-g}\left\{g^{\mu \nu} \partial_{\mu} \phi \partial_{\nu} \phi+\frac{1}{6} R \phi^{2}+\lambda \phi^{4}\right\}
$$


where $\lambda=e^{-4 \sigma_{0}} V\left(\frac{1}{6} e^{2 \sigma_{0}}\right)$. Thus we see that in a new variables the starting Hilbert-Einstein action (2) (remind that $\Phi$ is constant here) corresponds to the conformally coupled scalar field $\phi$. The extra scalar degree of freedom in (10) is compensated by extra symmetry local conformal invariance. Both theories are equivalent on the classical level. On quantum level the conformal invariance of the theory (10) will be probably broken because of the non-invariance of the measure of path integral over the metric (see [30] for the discussion of this point in Weyl gravity. Indeed it is not completely sufficient, and the conformal version should be investigated separately). Thus the new anomalous degree of freedom starts to propagate and the equivalence of two theories can be violated. Let us notice that the same frame for Einstein gravity has been recently used in [31] for the investigation of $2+\varepsilon$ quantum gravity.

And so, all the theories (四) can be divided into two sets. First set is labeled by $2 A B-$ $3\left(B_{1}\right)^{2}=0$, it is conformally equivalent to the General Relativity with cosmological constant. For the second set $2 A B-3\left(B_{1}\right)^{2} \neq 0$. Such models are conformally equivalent to (2) with non-constant $\Phi$. Below we shall deal only with the theories of the second type. On classical level the change of dynamical variables can be compensated by the change of the functions $A(\phi), B(\phi), C(\phi)$. However, as it was recently discussed by Magnano and Sokolowski [9], the natural choice of the frame is preferable from physical point of view already on classical level. One can face the same situation in the quantum theory as well. To see this, let us consider one interesting particular case [24]. If one put the potential term in (2) in the special way and make the shift of the field $\Phi=\phi-\phi_{0}$, where $\phi_{0}=$ const then the resulting theory

$$
S=\int d^{4} x \sqrt{-g}\left\{\frac{1}{4 \alpha} \phi^{2}+R\left(\phi-\phi_{0}\right)+\Lambda\right\}
$$

is equivalent to the special version of higher derivative quantum gravity

$$
S=\int d^{4} x \sqrt{-g}\left\{-\alpha R^{2}-\frac{1}{G} R+\Lambda\right\}
$$

However on quantum level it is so only if we do not introduce into the generating functional of the Green functions the external source for the auxiliary field $\phi$. If one make some nonlinear change of variables like the conformal transform described above, the auxiliary field and the conformal factor of the metric are mixed and we likely lose the simple relation between (12) and (11).

\section{The structure of the counterterms off and on mass shell}

The main purpose of the present paper is to investigate the theory (1) on quantum level within the one - loop approximation. The simple consideration based on power counting shows that the theory is non-renormalizable just as General Relativity. At the same time at one-loop order the last theory is renormalizable on mass shell [23]. This property holds even if the cosmological constant is included to the action [20] that enables one to apply some kind of renormalization group approach for it's study 16. That is why it looks interesting to consider the renormalization of our theory on mass shell. The next reason to do this is the gauge and parametrization independence of the effective action on mass shell. 
In this section we write down the classical equations of motion and the possible divergent structures, taking into account only the one loop order. Then we find some simple relations between counterterms and consider the divergent structures which are possible on shell. The equations of motion in the theory (11) have the form

$$
\begin{gathered}
B R^{\mu \nu}+g^{\mu \nu}\left[\left(B_{2}-\frac{A}{2}\right)(\nabla \phi)^{2}-\frac{R+C}{2}+B_{1}(\square \phi)\right]+\left(A-B_{2}\right)\left(\nabla^{\mu} \phi\right)\left(\nabla^{\nu} \phi\right)-B_{1}\left(\nabla^{\mu} \nabla^{\nu} \phi\right)=0 \\
B_{1} R+C_{1}-A_{1}(\nabla \phi)^{2}-2 A(\square \phi)=0
\end{gathered}
$$

Before going on to discuss the renormalization of the theory, one have to define the classical dimension of the field $\phi$. The form of the starting Lagrangian shows that there is some dimensional parameter $M$ from the very beginning. One can introduce such dimensional parameter in a different ways, that corresponds to different classical dimensions of the scalar field $\phi$. For instance, in the case of dimension-less $\phi$ the arbitrary functions $A, B, C$ include the dimensional parameter $M$ in a trivial way $A, B \sim M^{2}$ and $C \sim M^{4}$. On the contrary, if the dimension of $\phi$ is chosen as unity, then (if we want to consider arbitrary functions $A, B, C$ ), they depend on the ratio $\frac{\phi}{M}$. Of course the results of the explicit (one-loop in our case) calculations do not depend on this choice, and thus we can regard the dimension of $\phi$ according to our convenience. On this stage it is better to consider the dimension-less $\phi$. Then the arbitrary functions $A, B, C$ do not contain the dimensional parameter $M$. Another advantage of this choice is that $\phi$ and metric have an equal dimensions and therefore the power counting in a dilaton theory is essentially the same as compared with General Relativity.

If one is interested only in the one-loop divergences, then the counterterms contains the covariant terms of fourth order in derivatives. The most general action of this type has the form [25]:

$$
\begin{aligned}
\Gamma_{\text {div }}^{1-\text { loop }}=- & \frac{1}{16 \pi^{2}(n-4)} \int d^{4} x \sqrt{-g}\left[c_{w} C^{2}+c_{r} R^{2}+c_{4} R(\nabla \phi)^{2}+c_{5} R(\square \phi)+c_{6} R^{\mu \nu}\left(\nabla_{\mu} \phi\right)\left(\nabla_{\nu} \phi\right)+\right. \\
& \left.+c_{7} R+c_{8}(\nabla \phi)^{4}+c_{9}(\nabla \phi)^{2}(\square \phi)+c_{10}(\square \phi)^{2}+c_{11}(\nabla \phi)^{2}+c_{12}\right]+(\text { s.t. })
\end{aligned}
$$

where $C^{2}=C_{\mu \nu \alpha \beta} C^{\mu \nu \alpha \beta}$ is the square of Wyle tensor and $(\nabla \phi)^{2}=g^{\mu \nu} \nabla_{\mu} \phi \nabla_{\nu} \phi$. "s.t." means "surface terms". All $c_{w, r, 4, \ldots, 12}$ are some functions of $A(\phi), B(\phi), C(\phi)$ and their derivatives. One can easily check the following reduction formulas which show the surface form of the other possible structures [25].

$$
\begin{gathered}
c_{13}\left(\nabla^{\mu} R\right)\left(\nabla_{\mu} \phi\right)=-c_{13}^{\prime} R\left(\nabla_{\mu} \phi\right)^{2}-c_{13} R(\square \phi)+(\text { s.t. }) \\
c_{14} R_{\mu \nu}\left(\nabla^{\mu} \nabla^{\nu} \phi\right)=-c_{14}^{\prime} R_{\mu \nu}\left(\nabla^{\mu} \phi\right)\left(\nabla^{\nu} \phi\right)+\frac{1}{2} c_{14}^{\prime} R(\nabla \phi)^{2}+\frac{1}{2} c_{14} R(\square \phi)+(\text { s.t. }) \\
c_{15}\left(\nabla^{\nu} \phi\right)\left(\square \nabla_{\nu} \phi\right)=-c_{15}^{\prime}(\nabla \phi)^{2}(\square \phi)-c_{15}^{\prime}(\square \phi)^{2}+c_{15}^{\prime} R_{\mu \nu}\left(\nabla^{\mu} \phi\right)\left(\nabla^{\nu} \phi\right)+(\text { s.t. }) \\
c_{16}\left(\nabla_{\nu} \nabla_{\mu} \phi\right)^{2}=\frac{1}{2} c_{16}^{\prime \prime}(\nabla \phi)^{4}+\frac{3}{2} c_{16}^{\prime}(\nabla \phi)^{2}(\square \phi)+c_{16}(\square \phi)^{2}-c_{16} R_{\mu \nu}\left(\nabla^{\mu} \phi\right)\left(\nabla^{\nu} \phi\right)+(\text { s.t. }) \\
c_{17}\left(\nabla^{\nu} \square \nabla_{\nu} \phi\right)=c_{17}^{\prime \prime}(\nabla \phi)^{2}(\square \phi)+c_{17}^{\prime}(\square \phi)^{2}-c_{17}^{\prime} R_{\mu \nu}\left(\nabla^{\mu} \phi\right)\left(\nabla^{\nu} \phi\right)+(\text { s.t. }) \\
c_{18}\left(\square^{2} \phi\right)=c_{18}^{\prime \prime}(\nabla \phi)^{2}(\square \phi)+c_{18}^{\prime}(\square \phi)^{2}+(\text { s.t. }) \\
c_{19}(\square R)=c_{19}^{\prime \prime} R(\nabla \phi)^{2}+c_{19}^{\prime} R(\square \phi)+(\text { s.t. })
\end{gathered}
$$




$$
\begin{gathered}
c_{20}\left(\nabla_{\nu} \phi\right)\left(\nabla_{\mu} \phi\right)\left(\nabla^{\nu} \nabla^{\mu} \phi\right)=-\frac{1}{2} c_{20}^{\prime}(\nabla \phi)^{4}-\frac{1}{2} c_{20}(\nabla \phi)^{2}(\square \phi)+(\text { s.t. }) \\
c_{21}\left(\nabla^{\nu} \phi\right)\left(\nabla_{\nu} \square \phi\right)=-c_{21}^{\prime}(\nabla \phi)^{2}(\square \phi)-c_{21}(\square \phi)^{2}+(\text { s.t. })
\end{gathered}
$$

Here $c_{13, . ., 21}=c_{13, . ., 21}(\phi)$ are some (arbitrary) functions.

Thus the power counting consideration and the account of symmetries show that the possible counterterms have complicated form and differs from the classical action. Therefore the theory is expected to be non-renormalizable off shell. Let us now discuss the renormalization on mass shell. For this purpose we shall apply the equations of motion (13) and the reduction formulas (15) and rewrite the counterterms (14) and the classical action (11) in a maximally simple form. In particular, from the equations of motion (13) one can get the following relations

$$
\begin{gathered}
(\nabla \phi)^{2}=x R+y, \quad(\square \phi)=z R+w \\
\left(\nabla^{\mu} \nabla^{\nu} \phi\right)=r\left(\nabla^{\mu} \phi\right)\left(\nabla^{\nu} \phi\right)+s R^{\mu \nu}+t g^{\mu \nu} R+u g^{\mu \nu}
\end{gathered}
$$

where

$$
\begin{gathered}
x(\phi)=\frac{2 A B-3 B_{1}^{2}}{-2 A^{2}-3 A_{1} B_{1}+6 A B_{2}} \\
y(\phi)=\frac{4 A \mathrm{C}-3 B_{1} \mathrm{C}_{1}}{-2 A^{2}-3 A_{1} B_{1}+6 A B_{2}} \\
z(\phi)=\frac{-\left(B A_{1}\right)-A B_{1}+3 B_{1} B_{2}}{-2 A^{2}-3 A_{1} B_{1}+6 A B_{2}} \\
w(\phi)=\frac{2 \mathrm{C} A_{1}+A \mathrm{C}_{1}-3 B_{2} \mathrm{C}_{1}}{2 A^{2}+3 A_{1} B_{1}-6 A B_{2}} \\
r(\phi)=\frac{A-B_{2}}{B_{1}}, \\
t(\phi)=\frac{B A_{1} B_{1}+A B_{1}{ }^{2}-2 A B B_{2}}{2 B_{1}\left(-2 A^{2}-3 A_{1} B_{1}+6 A B_{2}\right)} \\
u(\phi)=\frac{2 A^{2} \mathrm{C}+\mathrm{C} A_{1} B_{1}-2 A \mathrm{C} B_{2}-A B_{1} \mathrm{C}_{1}}{2 B_{1}\left(2 A^{2}+3 A_{1} B_{1}-6 A B_{2}\right)}
\end{gathered}
$$

Next, combining the equations of motion (13) and the reduction formula for $c_{14}$ (15) we find

$$
\begin{aligned}
& \int d^{4} x \sqrt{-g} c_{6} R_{\mu \nu}\left(\nabla^{\mu} \phi\right)\left(\nabla^{\nu} \phi\right)=\int d^{4} x \sqrt{-g}\left[\frac{1}{2} c_{6} x R^{2}+\frac{1}{2} c_{6} R+\right. \\
& \left.+f(\phi)\left\{-s R_{\mu \nu}^{2}+\left(\frac{1}{2} r x+\frac{1}{2} z-t\right) R^{2}+\left(\frac{1}{2} r y+\frac{1}{2} w-u\right) R\right\}\right]
\end{aligned}
$$

where $f(\phi)$ is solution of the following differential equation

$$
f_{1}(\phi)+f(\phi) r(\phi)-c_{6}(\phi)=0
$$

Since the last equation have solution for any $r(\phi)$ and $c_{6}(\phi)$, we find that the on shell one loop divergences for our dilaton model (11) can be reduced to the form of higher derivative terms without explicit kinetic terms for the dilaton. Of course one can choose another basis and express everything in terms of dilaton structures only, removing the terms with higher powers of curvature. 


\section{One-loop calculations}

In this section we shall present the details of the calculation of the one-loop counterterms of the theory (1). For the purpose of calculation of the divergences we will apply the background field method and the Schwinger-De Witt technique. The features of the metric-dilaton theory do not lead to the necessity of some modifications of the calculational scheme, basically developed in the similar two-dimensional theory [27].

Let us start with the usual splitting of the fields into background $g_{\mu \nu}, \phi$ and quantum $h_{\mu \nu}, \varphi$ ones

$$
\phi \rightarrow \phi^{\prime}=\phi+\varphi, \quad g_{\mu \nu} \rightarrow g_{\mu \nu}^{\prime}+h_{\mu \nu}
$$

The one-loop effective action is given by the standard general expression

$$
\Gamma=\frac{i}{2} \operatorname{Tr} \ln \hat{H}-i \operatorname{Tr} \ln \hat{H}_{\text {ghost }}
$$

where $\hat{H}$ is the bilinear form of the action (再) with added gauge fixing term and $\hat{H}_{\text {ghost }}$ is the bilinear form of the gauge ghosts action. To perform the calculations in a most simple way one needs to introduce the special form of the gauge fixing term:

$$
S_{g f}=\int d^{4} x \sqrt{-g} \chi_{\mu} \frac{\alpha}{2} \chi^{\mu}
$$

where $\chi_{\mu}=\nabla_{\alpha} \bar{h}_{\mu}^{\alpha}+\beta \nabla_{\mu} h+\gamma \nabla_{\mu} \varphi, h=h_{\mu}^{\mu}, \bar{h}_{\mu \nu}=h_{\mu \nu}-\frac{1}{4} h g_{\mu \nu}$ and $\alpha, \beta, \gamma$ are some functions of the background dilaton, which can be tuned for our purposes. For instance, if one choose these functions as follows

$$
\alpha=-B, \quad \beta=-\frac{1}{4}, \quad \gamma=-\frac{B_{1}}{B}
$$

then the bilinear part of the action $S+S_{g f}$ and the operator $\hat{H}$ has especially simple (minimal) structure

$$
\begin{gathered}
\left(S+S_{g f}\right)^{(2)}=\int d^{4} x \sqrt{-g} \omega \hat{H} \omega^{T} \\
\hat{H}=\hat{K} \square+\hat{L}_{\rho} \nabla^{\rho}+\hat{M}
\end{gathered}
$$

Here $\omega=\left(\bar{h}_{\mu \nu}, h, \varphi\right)$ and $T$ means transposition. The components of $\hat{H}$ have the form

$$
\begin{aligned}
& \hat{K}=\left(\begin{array}{ccc}
\frac{B}{4} \delta^{\mu \nu \alpha \beta} & 0 & 0 \\
0 & -\frac{B}{16} & -\frac{B_{1}}{4} \\
0 & -\frac{B_{1}}{4} & \frac{B_{1}^{2}}{2 B}-A
\end{array}\right) \\
& \hat{L}^{\lambda}=\left(\begin{array}{ccc}
\frac{B_{1}}{4}\left(\delta^{\mu \nu \alpha \beta} g^{\tau \lambda}+2 g^{\nu \beta}\left(g^{\mu \tau} g^{\alpha \lambda}-g^{\alpha \tau} g^{\mu \lambda}\right)\right) & -\frac{B_{1}}{4} g^{\mu \tau} g^{\nu \lambda} & \left(\frac{B_{2}}{2}-A\right) g^{\mu \tau} g^{\nu \lambda} \\
\frac{B_{1}}{4} g^{\alpha \tau} g^{\beta \lambda} & -\frac{B_{1}}{16} g^{\tau \lambda} & \left(\frac{A}{4}-\frac{5}{8} B_{2}\right) g^{\tau \lambda} \\
\left(A-\frac{B_{2}}{2}\right) g^{\alpha \tau} g^{\beta \lambda} & \left(\frac{B_{2}}{8}-\frac{A}{4}\right) g^{\tau \lambda} & \left(\frac{B_{1}^{2}}{2 B}-A\right)_{1} g^{\tau \lambda}
\end{array}\right)\left(\nabla_{\tau} \phi\right)
\end{aligned}
$$




$$
\hat{M}=\left(\begin{array}{ccc}
\delta^{\mu \nu \alpha \beta}\left(\frac{B_{1}}{2}(\square \phi)+\left(\frac{B_{2}}{2}-\frac{A}{4}\right)(\nabla \phi)^{2}-\frac{C}{4}\right) & \frac{B_{2}}{2}\left(\nabla^{\mu} \nabla^{\nu} \phi\right) \\
+g^{\nu \beta}\left(-B_{1}\left(\nabla^{\mu} \nabla^{\alpha} \phi\right)+\left(A-B_{2}\right)\left(\nabla^{\mu} \phi\right)\left(\nabla^{\alpha} \phi\right)\right) & 0 & +\left(\frac{B_{3}}{2}-\frac{A_{1}}{2}\right)\left(\nabla^{\mu} \phi\right)\left(\nabla^{\nu} \phi\right) \\
+\frac{B}{4}\left(-\delta^{\mu \nu \alpha \beta} R+2 g^{\nu \beta} R^{\mu \alpha}+2 R^{\mu \alpha \nu \beta}\right) & -\frac{B_{1}}{2} R^{\mu \nu} & -\frac{3}{8} B_{2}(\square \phi) \\
\frac{B_{1}}{4}\left(\nabla^{\alpha} \nabla^{\beta} \phi\right)+\frac{B_{2}}{4}\left(\nabla^{\alpha} \phi\right)\left(\nabla^{\beta} \phi\right) & \frac{C}{16} & +\left(\frac{A_{1}}{8}-\frac{3}{8} B_{3}\right)(\nabla \phi)^{2} \\
& & +\frac{B_{1}}{8} R+\frac{C_{1}}{4} \\
A\left(\nabla^{\alpha} \nabla^{\beta} \phi\right)+\frac{A_{1}}{2}\left(\nabla^{\alpha} \phi\right)\left(\nabla^{\beta} \phi\right)-\frac{B_{1}}{2} R^{\alpha \beta} & -\frac{A}{4}(\square \phi) & -A_{1}(\square \phi) \\
& +\frac{A_{1}}{8}(\nabla \phi)_{1}^{2} & -\frac{A_{2}}{2}(\nabla \phi)^{2} \\
& & +\frac{C_{1}}{2} R+\frac{C_{2}}{2}
\end{array}\right)
$$

The next problem is to separate the divergent part of $\operatorname{Tr} \ln \hat{H}$. To make this we rewrite this trace in a following way.

$$
\operatorname{Tr} \ln \hat{H}=\operatorname{Tr} \ln \hat{K}+\operatorname{Tr} \ln \left(\hat{1} \square+\hat{K}^{-1} \hat{L}^{\mu} \nabla_{\mu}+\hat{K}^{-1} \hat{M}\right)
$$

One can notice that the first term does not give contribution to the divergences. Let us explore the second term which has standard minimal form and can be easily estimated with the use of standard Schwinger-DeWitt method [21, 23] (see also [22] for technical introduction and more complete references).

The bilinear form of the ghost action also has the minimal structure

$$
\hat{H}_{\text {ghost }}=g^{\mu \alpha} \square+\gamma\left(\nabla^{\alpha} \phi\right) \nabla^{\mu}+\gamma\left(\nabla^{\mu} \nabla^{\alpha} \phi\right)+R^{\mu \alpha}
$$

and it's contribution to the divergences can be easily derived with the use of the standard methods.

Summing up both contributions we find that the one-loop divergences have the form (14) that is in a full accord with the power counting consideration. The coefficient functions $c$ have the form

$$
\begin{gathered}
c_{w}=\frac{43}{120}-\frac{B_{1}{ }^{2}}{X}, \quad X=2 A B-3 B_{1}^{2} \\
c_{r}=\frac{1}{X^{2}}\left[\frac{1}{72}\left(76 A^{2} B^{2}-132 A B B_{1}{ }^{2}+171 B_{1}^{4}\right)-\frac{1}{6}\left(B\left(2 A B+9 B_{1}{ }^{2}\right) B_{2}\right)+\frac{1}{2}\left(B^{2} B_{2}{ }^{2}\right)\right] \\
c_{4}=\frac{1}{X^{3}}\left[\frac { 1 } { 2 4 B ^ { 2 } } \left(128 A^{4} B^{4}+8 A B^{5} A_{1}{ }^{2}-144 A^{2} B^{4} A_{1} B_{1}-1552 A^{3} B^{3} B_{1}{ }^{2}\right.\right. \\
\left.+36 B^{4} A_{1}{ }^{2} B_{1}{ }^{2}+312 A B^{3} A_{1} B_{1}{ }^{3}+5208 A^{2} B^{2} B_{1}{ }^{4}-6786 A B B_{1}{ }^{6}+3159 B_{1}{ }^{8}\right) \\
+\frac{B_{2}}{6 B}\left(-80 A^{3} B^{3}-6 B^{4} A_{1}{ }^{2}-24 A B^{3} A_{1} B_{1}+402 A^{2} B^{2} B_{1}{ }^{2}-54 B^{2} A_{1} B_{1}{ }^{3}-810 A B B_{1}{ }^{4}\right. \\
\left.\left.+459 B_{1}{ }^{6}\right)+2\left(2 A^{2} B^{2}+3 B^{2} A_{1} B_{1}+12 A B B_{1}{ }^{2}-9 B_{1}{ }^{4}\right) B_{2}{ }^{2}-6 A B^{2} B_{2}{ }^{3}\right] \\
c_{5}=\frac{1}{X^{2}}\left[\frac{1}{12 B}\left(4 A B^{3} A_{1}-128 A^{2} B^{2} B_{1}+18 B^{2} A_{1} B_{1}{ }^{2}+270 A B B_{1}{ }^{3}-225 B_{1}{ }^{5}\right)\right. \\
\left.+\frac{B_{2}}{2}\left(-2 B^{2} A_{1}+4 A B B_{1}+3 B_{1}{ }^{3}\right)\right] \\
c_{6}=\frac{1}{X^{2}}\left[\frac{B_{1}}{B^{2}}\left(8 A B^{3} A_{1}-4 A^{2} B^{2} B_{1}-6 B^{2} A_{1} B_{1}{ }^{2}+22 A B B_{1}{ }^{3}-15 B_{1}{ }^{5}\right)\right.
\end{gathered}
$$




$$
\begin{aligned}
& \left.+\frac{B_{2}}{B}\left(-8 A^{2} B^{2}+2 B^{2} A_{1} B_{1}+4 A B B_{1}{ }^{2}-3 B_{1}^{4}\right)-4 A B B_{2}{ }^{2}\right] \\
& c_{7}=\frac{1}{X^{2}}\left[\frac { 2 } { 3 B } \left(26 A^{2} B^{2} \mathrm{C}-85 A B C B_{1}{ }^{2}+63 \mathrm{C} B_{1}{ }^{4}+3 B \mathrm{C} B_{1}{ }^{2} B_{2}\right.\right. \\
& \left.\left.+8 A B^{2} B_{1} \mathrm{C}_{1}-6 B^{2} B_{1} B_{2} \mathrm{C}_{1}\right)+\frac{B \mathrm{C}_{2}}{6}\left(-2 A B-9 B_{1}^{2}+6 B B_{2}\right)\right] \\
& c_{8}=\frac{1}{32 B^{4} X^{4}}\left[2560 A^{6} B^{6}-448 A^{3} B^{7} A_{1}{ }^{2}+16 B^{8} A_{1}{ }^{4}+256 A^{4} B^{7} A_{2}\right. \\
& +2432 A^{4} B^{6} A_{1} B_{1}-22528 A^{5} B^{5} B_{1}{ }^{2}+1280 A^{2} B^{6} A_{1}{ }^{2} B_{1}{ }^{2}-1152 A^{3} B^{6} A_{2} B_{1}{ }^{2} \\
& -17120 A^{3} B^{5} A_{1} B_{1}{ }^{3}+96 B^{6} A_{1}{ }^{3} B_{1}{ }^{3}+81568 A^{4} B^{4} B_{1}{ }^{4}-880 A B^{5} A_{1}{ }^{2} B_{1}{ }^{4} \\
& +1856 A^{2} B^{5} A_{2} B_{1}{ }^{4}+42016 A^{2} B^{4} A_{1} B_{1}{ }^{5}-158592 A^{3} B^{3} B_{1}{ }^{6}+168 B^{4} A_{1}{ }^{2} B_{1}{ }^{6} \\
& -1248 A B^{4} A_{2} B_{1}{ }^{6}-43512 A B^{3} A_{1} B_{1}{ }^{7}+176976 A^{2} B^{2} B_{1}{ }^{8}+288 B^{3} A_{2} B_{1}{ }^{8} \\
& +16416 B^{2} A_{1} B_{1}{ }^{9}-108144 A B B_{1}{ }^{10}+28323 B_{1}{ }^{12}-2048 A^{5} B^{6} B_{2} \\
& +224 A^{2} B^{7} A_{1}^{2} B_{2}+128 A^{3} B^{7} A_{2} B_{2}+4480 A^{3} B^{6} A_{1} B_{1} B_{2}-15248 A^{2} B^{5} A_{1} B_{1}^{3} B_{2} \\
& +14208 A^{4} B^{5} B_{1}^{2} B_{2}-1408 A B^{6} A_{1}^{2} B_{1}^{2} B_{2}-448 A^{2} B^{6} A_{2} B_{1}^{2} B_{2}-192 B^{7} A_{1}^{3} B_{1} B_{2} \\
& -28416 A^{3} B^{4} B_{1}{ }^{4} B_{2}+744 B^{5} A_{1}{ }^{2} B_{1}{ }^{4} B_{2}+480 A B^{5} A_{2} B_{1}{ }^{4} B_{2}+13680 A B^{4} A_{1} B_{1}{ }^{5} B_{2} \\
& +9696 A^{2} B^{3} B_{1}{ }^{6} B_{2}-144 B^{4} A_{2} B_{1}{ }^{6} B_{2}-2628 B^{3} A_{1} B_{1}{ }^{7} B_{2}+21672 A B^{2} B_{1}{ }^{8} B_{2} \\
& -15444 B B_{1}{ }^{10} B_{2}+1792 A^{4} B^{6} B_{2}{ }^{2}+192 A B^{7} A_{1}{ }^{2} B_{2}{ }^{2}+60624 A^{2} B^{4} B_{1}{ }^{4} B_{2}{ }^{2} \\
& -19328 A^{3} B^{5} B_{1}{ }^{2} B_{2}{ }^{2}+576 B^{6} A_{1}{ }^{2} B_{1}{ }^{2} B_{2}{ }^{2}+7776 A B^{5} A_{1} B_{1}{ }^{3} B_{2}{ }^{2}-928 A^{2} B^{6} A_{1} B_{1} B_{2}{ }^{2} \\
& -6984 B^{4} A_{1} B_{1}{ }^{5} B_{2}{ }^{2}-69120 A B^{3} B_{1}{ }^{6} B_{2}{ }^{2}+25380 B^{2} B_{1}{ }^{8} B_{2}{ }^{2}+128 A^{3} B^{6} B_{2}{ }^{3} \\
& \left.-1152 A B^{6} A_{1} B_{1} B_{2}{ }^{3}-3072 A^{2} B^{5} B_{1}{ }^{2} B_{2}{ }^{3}+3888 B^{3} B_{1}{ }^{6} B_{2}{ }^{3}+576 A^{2} B^{6} B_{2}{ }^{4}\right] \\
& +\frac{B_{3}}{4 B^{2} X^{2}}\left[-10 A B^{3} A_{1}-24 A^{2} B^{2} B_{1}+19 B^{2} A_{1} B_{1}{ }^{2}+36 A B B_{1}{ }^{3}\right. \\
& \left.-6 B_{1}^{5}+8 A B^{2} B_{1} B_{2}-36 B B_{1}^{3} B_{2}\right] \\
& c_{9}=\frac{1}{8 B^{3} X^{3}}\left[-16 A^{3} B^{5} A_{1}+8 B^{6} A_{1}^{3}+576 A^{4} B^{4} B_{1}-16 A B^{5} A_{1}{ }^{2} B_{1}\right. \\
& +160 A^{2} B^{4} A_{1} B_{1}{ }^{2}-2960 A^{3} B^{3} B_{1}{ }^{3}+12 B^{4} A_{1}{ }^{2} B_{1}{ }^{3}-324 A B^{3} A_{1} B_{1}{ }^{4} \\
& +5400 A^{2} B^{2} B_{1}{ }^{5}+90 B^{2} A_{1} B_{1}{ }^{6} \cdot-4176 A B B_{1}{ }^{7}+1107 B_{1}{ }^{9} \\
& +56 A^{2} B^{5} A_{1} B_{2}+960 A^{3} B^{4} B_{1} B_{2}-48 B^{5} A_{1}{ }^{2} B_{1} B_{2}-144 A B^{4} A_{1} B_{1}{ }^{2} B_{2} \\
& -4032 A^{2} B^{3} B_{1}{ }^{3} B_{2}+234 B^{3} A_{1} B_{1}{ }^{4} B_{2}+6228 A B^{2} B_{1}{ }^{5} B_{2}-3186 B B_{1}{ }^{7} B_{2} \\
& \left.+48 A B^{5} A_{1} B_{2}{ }^{2}-240 A^{2} B^{4} B_{1} B_{2}{ }^{2}+216 A B^{3} B_{1}{ }^{3} B_{2}{ }^{2}-108 B^{2} B_{1}{ }^{5} B_{2}{ }^{2}\right]-\frac{B_{3}}{B} \\
& c_{10}=\frac{1}{8 B^{2} X^{2}}\left[4 B^{3} A_{1}\left(B A_{1}-4 A B_{1}\right)+12 B^{2} B_{1}^{2}\left(18 A^{2}-A_{1} B_{1}\right)+B_{1}^{4}\left(387 B_{1}^{2}-528 A B\right)\right]-\frac{B_{2}}{B} \\
& c_{11}=\frac{1}{4 B^{3} X^{3}}\left[\left(64 A^{4} B^{4}+16 A^{2} B^{4} A_{1} B_{1}-832 A^{3} B^{3} B_{1}{ }^{2}-40 B^{4} A_{1}{ }^{2} B_{1}{ }^{2}\right.\right. \\
& +16 A B^{4} A_{2} B_{1}{ }^{2}+40 A B^{3} A_{1} B_{1}{ }^{3}+3176 A^{2} B^{2} B_{1}{ }^{4}-24 B^{3} A_{2} B_{1}{ }^{4}-24 B^{2} A_{1} B_{1}{ }^{5}
\end{aligned}
$$




$$
\begin{aligned}
& -4356 A B B_{1}{ }^{6}+2070 B_{1}{ }^{8}-96 A^{3} B^{4} B_{2}+40 A B^{4} A_{1} B_{1} B_{2}+160 A^{2} B^{3} B_{1}{ }^{2} B_{2} \\
& \left.+84 B^{3} A_{1} B_{1}{ }^{3} B_{2}-420 A B^{2} B_{1}{ }^{4} B_{2}+234 B B_{1}{ }^{6} B_{2}-72 A B^{3} B_{1}{ }^{2} B_{2}{ }^{2}+36 B^{2} B_{1}{ }^{4} B_{2}{ }^{2}\right) \mathrm{C} \\
& +\left(-16 A^{2} B^{5} A_{1}+96 A^{3} B^{4} B_{1}+80 B^{5} A_{1}^{2} B_{1}-32 A B^{5} A_{2} B_{1}-56 A B^{4} A_{1} B_{1}{ }^{2}\right. \\
& -808 A^{2} B^{3} B_{1}{ }^{3}+48 B^{4} A_{2} B_{1}{ }^{3}-24 B^{3} A_{1} B_{1}{ }^{4}+1140 A B^{2} B_{1}{ }^{5} \\
& -612 B B_{1}^{7}-40 A B^{5} A_{1} B_{2}+224 A^{2} B^{4} B_{1} B_{2}-228 B^{4} A_{1} B_{1}^{2} B_{2} \\
& \left.+108 A B^{3} B_{1}{ }^{3} B_{2}+54 B^{2} B_{1}{ }^{5} B_{2}+120 A B^{4} B_{1} B_{2}{ }^{2}-36 B^{3} B_{1}{ }^{3} B_{2}{ }^{2}\right) \mathrm{C}_{1} \\
& +\left(64 A^{3} B^{5}-20 B^{6} A_{1}{ }^{2}+8 A B^{6} A_{2}-16 A B^{5} A_{1} B_{1}-112 A^{2} B^{4} B_{1}{ }^{2}\right. \\
& -12 B^{5} A_{2} B_{1}{ }^{2}+60 B^{4} A_{1} B_{1}{ }^{3}+120 A B^{3} B_{1}{ }^{4}-45 B^{2} B_{1}{ }^{6}-16 A^{2} B^{5} B_{2} \\
& \left.\left.+72 B^{5} A_{1} B_{1} B_{2}-48 A B^{4} B_{1}{ }^{2} B_{2}-72 B^{3} B_{1}{ }^{4} B_{2}-24 A B^{5} B_{2}{ }^{2}\right) \mathrm{C}_{2}\right] \\
& +\frac{\mathrm{C}_{3}}{2 X^{2}}\left[2 B^{2} A_{1}-4 A B B_{1}-3 B_{1}^{3}\right] \\
& c_{12}=\frac{1}{X^{2}}\left[\frac { 1 } { B ^ { 2 } } \left(20 A^{2} B^{2} \mathrm{C}^{2}-56 A B \mathrm{C}^{2} B_{1}{ }^{2}+41 \mathrm{C}^{2} B_{1}{ }^{4}-8 A B^{2} \mathrm{C}_{1} \mathrm{C}_{1}+\frac{B^{2} \mathrm{C}_{2}{ }^{2}}{2}\right.\right. \\
& \left.\left.+4 A B^{3} \mathrm{C}_{1}{ }^{2}+2 B^{2} B_{1}{ }^{2} \mathrm{C}_{1}{ }^{2}\right)+2 B_{1}\left(\mathrm{C} B_{1}-2 B \mathrm{C}_{1}\right) \mathrm{C}_{2}+4 B \mathrm{C} B_{1}{ }^{3} \mathrm{C}_{1}\right]
\end{aligned}
$$

Let us now make some comments concerning the above result. The one loop divergences (14), (27) essentially depend on the choice of the functions $A(\phi), B(\phi), C(\phi)$ in the starting action (1). In particular, this dependence concerns the $c_{w}$ and $c_{r}$ functions, which correspond to the terms with the second powers in curvature tensor. The above expressions are valid only in the case $X=2 A B-3 B_{1}^{2} \neq 0$. For $X=0$ the calculational scheme must be modified because of extra conformal symmetry. In this case one has to introduce the additional gauge fixing condition for conformal symmetry. It is easy to see that if such condition is taken in the form $h=0$ then the degeneracy of $\hat{K}$ is removed.

The curvature squared terms in (27) are in a good accord with the same terms calculated earlier in [23]. The direct comparison of (27) with the results of other authors is difficult since they have used different choice of quantum variables.

\section{One-loop finiteness and renormalizability on shell}

And so we observe that the one-loop divergences in the theory under discussion have rather complicated form, and include all possible structures of the action (14). In this respect the theory is similar to General Relativity, where all possible counterterms also appear [23]. It is well known that in the last case all the counterterms disappear on mass shell. Therefore it is interesting to consider the divergences (27) when the equations of motion (13) are taken into account. Then, with the use of (17) and (18) we obtain the one loop divergences in the form

$$
\Gamma_{\text {on-shell,div }}^{1-\text { loop }}=-\frac{1}{\epsilon} \int d^{4} x\left[k_{w} C^{2}+k_{r r} R^{2}+k_{r} R+k_{l}\right]
$$

where $\epsilon=(4 \pi)^{2}(n-4)$ and the values of coefficients are

$$
k_{l}(\phi)=c_{12}+c_{10} w^{2}+c_{11} y+c_{9} w y+c_{8} y^{2}
$$




$$
\begin{gathered}
k_{r}(\phi)=c_{7}-f u+c_{5} w+\frac{f w}{2}+c_{11} x+c_{9} w x+ \\
+c_{4} y+\frac{c_{6} y}{2}-\frac{f r y}{2}+2 c_{8} x y+2 c_{10} w z+c_{9} y z \\
k_{r r}(\phi)=c_{r}-\frac{f s}{3}-f t+c_{4} x+\frac{c_{6} x}{2}-\frac{f r x}{2}+c_{8} x^{2}+c_{5} z+\frac{f z}{2}+c_{9} x z+c_{10} z^{2} \\
k_{w}(\phi)=c_{w}-\frac{f s}{2}
\end{gathered}
$$

and $x, y, z, w, t, u, f$ have been defined in (17), (18). It is very important that the values of $k_{w, r r, r, l}$ do not depend on the choice of gauge fixing. From this follows that the finite solutions which we find below are independent on gauge and therefore good defined.

The divergences in the higher derivative dimension-less sector do not depend on the dimensional function $C(\phi)$ and therefore can be analyzed independently on the others. Let us start the search of finite solutions with the higher derivative structures and consider the equations $k_{w}=o$ and $k_{r r}=0$. In order to find the solutions we must solve these differential equations that looks extremely difficult problem. However one can see that the divergent coefficients (27) possess some homogeneouty. Taking this into account one can successfully find the finite solutions. In fact we have found three finite solutions of power type

$$
A(\phi)=a \phi^{m}, \quad B(\phi)=b \phi^{m+2}
$$

with different real values of $m$ and of the ratio $\frac{a}{b}$.

$$
\begin{gathered}
\left(m_{1}, \frac{a_{1}}{b_{1}}\right)=(-23.4851 \ldots, 1413.45 \ldots), \\
\left(m_{2}, \frac{a_{2}}{b_{2}}\right)=(-0.526300 \ldots, 2.72924 \ldots), \\
\left(m_{3}, \frac{a_{3}}{b_{3}}\right)=(-0.317820 \ldots, 4.09345 \ldots)
\end{gathered}
$$

The conditions $k_{w}=o$ and $k_{r r}=0$ do not fix the values of the constants $a, b$ but only their ratio. Let us notice that the algebraic equation for $m$ is of fifth order and therefore the fact of existence of real solution is independent of the numerical details in the expression (27). Thus we have found the form of the functions $A(\phi)$ and $B(\phi)$ for which our dilaton model without cosmological term $C(\phi)$ is finite on shell. Note that $C(\phi)$ term doesn't give contributions to the higher derivative counterterms due to it's dimension. However, if this term is included, situation becomes a little bit more complicated.

If we substitute the expressions (30) into the classical action (11), then the term, linear in curvature, is equal to zero. Thus the theory under consideration can be renormalizable on shell only if $k_{r}=0$. Therefore we must choose $C(\phi)$ in such a way that the counterterm, linear in curvature, is lacking on shell.

If we choose $C(\phi)=c \phi^{2(m+2)}$ (that corresponds to the same power of $\phi$ in both $C(\phi)$ and counterterm) then the classical action of the theory has the form

$$
S=\int d^{4} x \sqrt{-g}\left[-c \phi^{2(m+2)}\right]
$$


and the on shell divergences are

$$
\Gamma_{\text {on-shell,div }}^{1-\text { loop }}=-\frac{1}{16 \pi^{2}(n-4)} \int d^{4} x\left[s_{1} \frac{c}{b} \phi^{m+2} R+s_{2} \frac{c^{2}}{b^{2}} \phi^{2(m+2)}\right]
$$

where $s_{1}, s_{2}$ have the approximate numerical values (here and below we omit dots)

$$
\left(s_{1}, s_{2}\right)=(1.45386,0.05772), \quad(-0.55182,-3.95364), \quad(-0.105262,-3.06052)
$$

for the solutions (31)-(33) correspondingly. Thus for this choice of $C(\phi)$ the theory is not renormalizable even on mass shell. Hence we have to look for another values of the power.

Substituting (30) into the expression for $k_{r}$ we obtain the ordinary differential equation for $C(\phi)$.

$$
\lambda_{0} \phi^{3} C_{3}+\lambda_{1} \phi^{2} C_{2}+\lambda_{2} \phi C_{1}+\lambda_{3} C=0
$$

Here the indices stand for the derivatives with respect to $\phi$ (9)). For three versions of $\left(m, \frac{a}{b}\right)$ given in (31), (32), (33) correspondingly, the constants $\lambda_{0,1,2,3}$ have the following values:

$$
\begin{array}{cccc}
\lambda_{0}=-2.49128, & \lambda_{1}=-813.35, & \lambda_{2}=-6170.03, & \lambda_{3}=1310940 \\
\lambda_{0}=1.25083, & \lambda_{1}=-7.41961, & \lambda_{2}=13.6904, & \lambda_{3}=-4.71357 \\
\lambda_{0}=2.94653, & \lambda_{1}=-23.1234, & \lambda_{2}=60.081, & \lambda_{3}=-50.1804
\end{array}
$$

The equations (36) can be easily solved in the form

$$
C_{i}(\phi)=L_{i 1} \phi^{k_{i 1}}+L_{i 2} \phi^{k_{i 2}}+L_{i 3} \phi^{k_{i 3}}
$$

where $C_{i}(\phi)$ corresponds to $m_{i}$ and $L_{11,12, \ldots, 33}$ are arbitrary integration constants and the following powers correspond to coefficients (37), (38), (39)

$$
\begin{aligned}
& k_{11}=35.4101, \quad k_{12}=-47.7636, \quad k_{13}=-311.125 \\
& k_{21}=5.77717, \quad k_{22}=0.222461, \quad k_{23}=2.93212 \\
& k_{31}=3.36417, \quad k_{32}=0.752027, \quad k_{33}=6.73149
\end{aligned}
$$

The above solutions give the form of the function $C(\phi)$ which provide the absence of the non-renormalizable $k_{r} R$ type counterterm. The only structures which survive on shell in both classical action and counterterms are the potential ones. In this respect the theory under consideration is sharing the corresponding property of the Einstein gravity, where the only cosmological term remains when one uses the equations of motion. In the Einstein gravity with cosmological term this leads to the renormalizability of the theory on shell and enables one to consider the renormalization group equation for the cosmological constant.

Let us do the same and consider the renormalization of the potential function $C(\phi)$. We consider only the simple case of $C(\phi)=L \phi^{k}$ that is take two of $L$ 's equal to zero, and the third arbitrary. The renormalization of the potential function follows from (30), (29), (27) and has the form

$$
L^{(0)}\left(\phi^{(0)}\right)^{k}=\mu^{n-4}\left[L \phi^{k}+\frac{1}{\varepsilon} \frac{Q L^{2}}{b^{2}} \phi^{2 k-2 m-4}\right]
$$


Here we have included the factors of dimensional parameter $\mu$ related with the use of dimensional regularization. $Q$ is some number, which depends on the ratio of $a$ and $b$. The values of $Q$ which correspond to the $k$ 's from (41) - (43) and $m$ 's from (31) are

$$
\begin{array}{lrlrl}
Q_{11} & =10.2275, & Q_{12}=-1.51342, & Q_{13}=1249.04 \\
Q_{21}=19.7562, & Q_{22}=26.9177, & Q_{23}=-1.39468 \\
Q_{31}=-1.42338, & Q_{32}=268.66, & Q_{33}=664.993
\end{array}
$$

It is easy to see that the potential type divergences can not be removed by the transformation of $L$ but only by the following renormalization transformation of scalar field $\phi$.

$$
\phi^{(0)}=\mu^{\frac{n-4}{k}}\left[\phi+\frac{1}{\varepsilon} \frac{Q(a / b) L}{k b^{2}} \phi^{k-2 m-3}\right]
$$

The relation (44) does not enable us to find the dimensions of both $L$ and $\phi$. Since all three terms in (44) have (if $n=4$ ) dimension 4 , we can easily obtain two equations for dimensions $d_{L}, d_{b}$ (dimension of constant $b$ ) and $d_{\phi}$.

$$
\begin{gathered}
d_{L}+k d_{\phi}=4 \\
d_{L}+(k-m-2) d_{\phi}-d_{b}=2
\end{gathered}
$$

Another equation for these dimensions comes from the starting action

$$
d_{b}+(m+2) d_{\phi}=2
$$

together with $d_{a}=d_{b}$. However the last three equations are dependent and thus we only can express the dimensions of $a, b, L$ via $d_{\phi}$.

The renormalization relation (48) together with (49) and (50) enables us to explore the renormalization group equations for the effective charges. he renormalization group function for the scalar field $\phi$ is defined in a standard way and has the form

$$
\gamma_{\phi}=\mu \frac{d \phi}{d \mu}=-\frac{n-4}{k} \phi+\frac{1}{(4 \pi)^{2}} \frac{Q(a / b) L}{b^{2} k^{2}}(k-2 m-4) \phi^{k-2 m-3}
$$

Since we consider the four dimensional theory the first term in the rhs can be omitted and thus we arise at the following renormalization group equations for $L(t)$ and $\phi(t)$

$$
\begin{gathered}
(4 \pi)^{2} \frac{d L}{d t}=\left(k d_{\phi}-4\right) L \\
(4 \pi)^{2} \frac{d \phi}{d t}=\frac{Q(a / b) L}{b^{2} k^{2}}(k-2 m-4) \phi^{k-2 m-3}-d_{\phi} \phi
\end{gathered}
$$

Indeed one can easily write the similar equations for $a(t)$ and $b(t)$. Since these constants are not renormalized, their values depend on scale only due the classical dimensions. Since all these dimensions are ill defined the equations looks rather artificial. The only way to extract some information is to consider the quantities with definite dimension.

One can see that in the theory under consideration all the on shell divergences can be removed by the renormalization of the dilaton field. As a result there are the nontrivial 
renormalization group equations for $\phi$ which acquire the anomalous dimension. The scale dependence of the parameters $a, b, L$ and the field $\phi$ leads to the effective running of the cosmological constant. To see this let us consider the effective potential of the scalar field. Upon the normalization conditions are introduced, the effective potential has the form

$$
V_{\text {eff }}=\sum\left\{b \phi^{m+2} R\left[1+(m+2) \gamma \ln \frac{M_{i}(\phi)}{\mu}\right]+L \phi^{k}\left[1+k \gamma \ln \frac{M_{i}(\phi)}{\mu}\right]\right\}
$$

where $M_{i}(\phi)$ are the effective masses, which are the eigenvalues of the operators (24) and (26) and the algebraic summation is performed according to rule (21). $\mu$ is the dimensional parameter of renormalization, and $\gamma$ is the renormalization group function for dilaton (51). Since we are interested in the qualitative scaling behavior of the Newtonian and cosmological constants the logarithmic corrections in (53) are not relevant and one can deal with the renormalization group improved classical potential of the theory.

$$
V_{i m p}=b(t) \phi^{m+2}(t) R+L(t) \phi^{k}(t)
$$

One can easily see that for some of the solutions (41) - (43), (31) - (33) for the potential (54) it is satisfied the criteria of the second order phase transition

$$
\frac{d V_{i m p}}{d \phi}=0, \quad \frac{d^{2} V_{i m p}}{d \phi^{2}}>0
$$

The first of (55) leads to the relation between critical values of scalar field and curvature

$$
R_{c}=-\frac{L k}{b(m+2)} \phi_{c}^{k-m-2}
$$

and the second impose the restriction $k-m>2$. The last condition is satisfied for the models with $k_{11}, k_{21}, k_{23}, k_{31}, k_{33}$ (41) - (43) and corresponding values of $m$ in (31) - (33). Note that the stability of vacuum at classical level require positive $k, m+2, b$ and negative $L$. Thus the value of $k_{11}$ is not compatible with physical requirements. However another four models are and thus our theory allow the second order phase transitions. In the point of minima $\phi_{0}$ the renormalization group improved classical potential has the form of the Hilbert - Einstein action

$$
S_{\text {min }}=\int d^{4} x \sqrt{-g}\left\{-\frac{1}{G_{\text {ind }}} R+\Lambda_{\text {ind }}\right\}
$$

where the induced values of Newtonian $G_{\text {ind }}$ and cosmological $\Lambda_{\text {ind }}$ constants are defined as $G_{i n d}^{-1}=b \phi_{0}^{m+2}$ and $\Lambda_{i n d}=L \phi_{0}^{k}$. Since the parameters $b$ and $L$ are not renormalized the scaling dependence of $G_{i n d}$ and $\Lambda_{i n d}$ is caused by the only renormalization of dilaton (48) and by the classical dimension of the constants, which are well defined in the case. Since the classical dimension will surely dominate in the renormalization group equations for $G_{i n d}$ and $\Lambda_{i n d}$, it is reasonable to rewrite the renormalization group equation (52) in terms of dimension-less parameter $\eta(t)=\Lambda_{\text {ind }}(t) G_{i n d}^{2}(t)$. The equation for $\eta(t)$ has remarkably simple form

$$
(4 \pi)^{2} \frac{d \eta}{d t}=\frac{(k-2 m-4)^{2}}{k^{2}} Q(a / b) \eta^{2}
$$


which indicate to the standard asymptotical behavior of this parameter. And so the scaling behaviour of the induced cosmological constant is defined by the sign of the number $Q(a / b)$. One can see from (45) - (3.231) that for the physically relevant solutions with $k_{21}, k_{33}$ from (42), (43) the value $Q$ is positive. In these models the effective cosmological constant decrease at low energies. Thus we observe that the quantum effects in these versions of theory under consideration describe the vanishing of the cosmological constant in far IR. As a result the observable low energy value of the cosmological constant is small as compared with the high energy value. On the countrary, for $k_{23}, k_{31}$ (42), (43) the sign of $Q$ is negative and the effective cosmological constant decrease at high energy scale.

And so we have found that the model (1) leads to the effective running of the cosmological constant if the last is measured in the units of the Newtonian constants. Earlier the running of these couplings has been in the framework of Higher derivative models [32], in the gauge models on external classical background [33] and also in the higher derivative dilaton model [25]. The interesting property of our model is that here we observe qualitatively different asymptotical regims. In this respect the theory of one loop quantum dilaton gravity share the features of the higher derivative dilaton model [25].

\section{$6 \quad$ Interaction with matter fields}

In the previous sections we have shown there are some special versions of the general dilaton model (11) which are renormalizable on shell at one - loop level. In spite of that the expression for counterterms (27) is very cumbersome (one can truly say terrible) all those counterterms vanish on shell if the starting functions are chosen in a special way. Thus the special cases (27) - (27), (27) - (43) are sharing the same property of quantum Einstein gravity (with cosmological constant), which is also renormalizable on shell [23, 20]. However in the last case the interaction with matter fields leads to the violation of one loop on shell renormalizability [34. In this section we show that for our dilaton model it is also the case. If the matter fields are included the arbitrary functions $A(\phi), B(\phi)$ can not be fine tuned in such a way that the on shell higher derivative counterterms vanish.

Below we consider this in some details. One can suppose for simplicity that the matter fields action is composed by the vector fields only, and that the action of matter fields has the standard form and matter fields do not interact with the dilaton field $\phi$ directly, but

only via metric. Thus we consider the most simple case which corresponds to the first work in [34] when the gravity was described by the Hilbert - Einstein action. According to [34] he renormalizability of the Einstein - Maxwell system is violated by the counterterms like $T_{\mu \nu} T^{\mu \nu}$ and $R_{\mu \nu} T^{\mu \nu}$. Here $T_{\mu \nu}$ is the Energy - Momentum Tensor of the matter

$$
T_{\mu \nu}=-\frac{2}{\sqrt{-g}} \frac{\delta S_{m}}{\delta g^{\mu \nu}}
$$

which is traceless in four dimensions $T_{\mu \nu} g^{\mu \nu}=0$ that reflects the conformal invariance property of the matter fields action.

The contributions of matter fields and the ones of "mixed sector" to the one loop counterterms in our metric - dilaton gravity (after the renormalization in a matter fields sector, however for vectors it is not necessary [34]) lead to the following change of the counterterms. The general form of the divergences (14) is changed because of $T_{\mu \nu} T^{\mu \nu}$ and $R_{\mu \nu} T^{\mu \nu}$ terms, 
and moreover coefficient $c_{w}$ acquire the addition

$$
c_{w} \rightarrow c_{w}+\frac{N_{v}}{10}+\frac{N_{f}}{20}
$$

The contributions to $c_{r}$ are forbidden by conformal invariance (see, for example, [22]) and others are lacking because the matter fields decouple from dilaton.

Let us now discuss the on shell renormalization which is a little bit more complicated. The first of the classical equations of motion (13) acquire the additional term $\frac{1}{2} T_{\mu \nu}$ on the rhs. However the second equation (13) remains unchanged as well as the trace of the first one. The transfer on shall is performed with the use of the formulas (16) - (19). Since (16) and (17) are based just on the second equation (13) and on the the trace of the first one, we conclude that for the theory with matter (16) and (17) are the same as in a pure metric dilaton gravity. The detailed analysis show that the equation (19) is also the same. However, the expression (18) changes according to

$$
s R_{\mu \nu} R^{\mu \nu} \rightarrow s R_{\mu \nu} R^{\mu \nu}-\frac{1}{2 B_{1}(\phi)} R^{\mu \nu} T_{\mu \nu}
$$

Therefore there is following contribution of the matter fields sector to the on shell divergences

$$
\delta \Gamma=-\frac{1}{2 \varepsilon} \int d^{4} x \sqrt{-g} \frac{f(\phi)}{B_{1}(\phi)} R^{\mu \nu} T_{\mu \nu}
$$

which has to be added to (28) together with original $T_{\mu \nu} T^{\mu \nu}$ and $R_{\mu \nu} T^{\mu \nu}$ terms. Moreover one must take into account the numerical change in $k_{w}$ which corresponds to (59) and (29).

Thus if we try to cancel the higher derivative on shell divergences by choosing the functions $A$ and $B$ we face the more difficult problem than we have met in Section 5 . As it was already pointed out there, the equations $k_{w}=k_{r r}=0$ have real solutions and therefore the $R^{2}$ type counterterms can be removed. However the $T_{\mu \nu} T^{\mu \nu}$ and $R_{\mu \nu} T^{\mu \nu}$ structures survive and we already do not have free parameters to cancel them. Thus one can observe that the on shell renormalizability is lacking in our metric - dilaton theory just as in purely metric gravity [34]. Moreover the renormalizability is violated by exactly the same two structures which are related with the traceless Energy - Momentum Tensor of the matter fields.

\section{Two special cases}

In this section we briefly discuss two special cases of the theory (1) which are of special physical interest. One can consider this part of the paper as some kind of quantum gravity phenomenology.

i) Let us consider the theory (11) that is classically equivalent to the special version of higher derivative quantum gravity (12). The transfer to quantum theory can be performed by introducing the generating functional of Green functions. If one introduce the external source for the auxiliary scalar field $\phi$, then the direct link between two models (12) and (11) will be lost. Therefore if we like to have such a link, the external source must be introduced for the metric only. If we consider the effective action in a background gauge, then the lack of external source for scalar corresponds to the lack of the background scalar field. In this case the only counterterms in the theory are the ones of the $c_{w}, c_{r}, c_{7}, c_{12}$ type in (14) and 
only one of them, namely $c_{w}$, violate the renormalizability. If we restrict ourselves by only the conformally flat background, then the theory is renormalizable and we can construct the renormalization group equations for the effective couplings $\alpha(t), G(t), \Lambda(t)$. The study of these equations show that the theory possesses cosmologically acceptable regime and, in particular, the quantum effects lead to exponential decrease of $\alpha(t)$ and $\Lambda(t)$ at high energies [24]. Indeed the additional restriction on the background metric is not completely consistent from formal point of view. On this way we remove the divergent diagrams with massive spin two particles by hands. On the other side, in higher derivative gravity the existence of these particles (which have the wrong sign of the kinetic term and thus are unphysical) lead to the well known unitarity problem, so their removal here is not much worst then the existence. The detailed analysis of the renormalization group equations in the model (12) and their cosmological consequences will be given in [24].

ii) Another interesting particular case is related with another extra condition on the background. One can suppose that the background scalar field is varying slowly as compared with the metric, and remove all the terms with the derivatives of scalar. Then we find that the only types of the counterterms which survive are the same as in the previous case. The next natural step is to look for the solutions of the equations $c_{w}=c_{r}=0$ and so construct the theory with renormalizable potential. We have explored the (30) form of the functions $A(\phi)$ and $B(\phi)$. It turned out that the equations for $a, b, m$ following from $c_{w}=c_{r}=0$ condition, do not have real solutions, and hence this idea doesn't work. At the same time the above equations for $A(\phi)$ and $B(\phi)$ are ill defined because the conditions $c_{w}=c_{r}=0$ depend on the choice of gauge fixing parameters. The possible way to remove this dependence is related with the use of the Vilkovisky's unique effective action [35] which coincide with the conventional effective action on shell, but differs off shell. Within this scheme we shall get the equations for $A(\phi)$ and $B(\phi)$ which have the structure similar to above but with different numerical coefficients. One can suppose that taking into account the Vilkovisky corrections to the divergences, we can get the real solutions and to construct the dilaton theory with renormalizable potential.

\section{Discussion}

In this paper we have considered the different aspects of the one-loop renormalization in the theory (1). We have shown that the models of this type can be divided into two classes models of one are conformally equivalent to the general relativity and also to the conformally coupled to gravity scalar field. The models of second class are conformally equivalent to the model (2) with non-constant $\Phi$, and any model of this type can be related to another one by some change of variables together with some change of potential function.

The one-loop calculations have been carried out for the general model (四) in original variables, with the use of background field method and some calculational improvements basically introduced in similar $d=2$ theory. Our calculational method does not need the conformal transformation of the metric and therefore is applicable (with minor standard modifications) to the higher derivative dilaton model which has been recently formulated in [25, 30]. The theory under consideration leads to a very cumbersome divergences and hence is non-renormalizable in usual sense. At the same time if the cosmological (or potential) term $C(\phi)$ is lacking then the theory with the fine tuned functions $A(\phi)$ and $B(\phi)$ is finite on 
classical equations of motion, that is possesses the same property as general relativity. If the potential term $C(\phi)$ is included the theory is renormalizable on shell, and the divergences can be removed by the renormalization of the dilaton field. Summing up, we have constructed 9 versions of the model (11) with

$$
A(\phi)=a \phi^{m}, \quad B(\phi)=b \phi^{m+2} \quad C(\phi)=L \phi^{k}
$$

with $\frac{a}{b}, m, k$ defined in (33) - (33), (41) - (43). All these versions have qualitatively the same renormalization property as Einstein gravity with cosmological term. They are nonrenormalizable off shell and renormalizable on shell. If the matter fields are included, then the on shell renormalizability is lost. The higher loops are expected to violate the on shell renormalizability because of appearance of the counterterms with third powers of curvature. The one loop renormalizability of the theory enable us to apply the renormalization group method for it's study. It turns out that the effective potential of the theory indicate to the possibility of the second order phase transitions and in the point of minima the potential has the form of the Hilbert - Einstein action with both Newtonian and cosmological constants depending on scale. It is important that the results of our analysis are independent on the choice of gauge fixing condition because we consider the on shell renormalization (see [36] for the most complete investigation of the gauge dependence in quantum field theory.

The one-loop calculations in the model (1) have been recently published in [37. In this paper, by use of transformation like (3) (the special form of these transformations had been originally introduced in [23] for this purposes) the general model is reduced to the special case with $A=-\frac{1}{2}, B=$ const, and then the divergences are calculated in a special variables which correspond to this reduced model. We have performed the calculations in an original variables, and in this sense our result differs from the one of [37]. In particular, the use of original variables allows the direct application to the model (11). Next, if considered off shell, our counterterms differ from the ones, derived in [37 because of different choice of quantum variables. This difference indicate to the parametrization dependence of all the counterterms. As a consequence, the generalized beta functions, which have been derived in [37, are likely to be parametrization (and probably gauge) dependent. This fact is a direct consequence of the non-renormalizability of the theory in standard sense.

The calculation in original variables is especially important for the study of $R+R^{2}$-gravity. On quantum level this model is equivalent to some version of (1), but only in original field variables, since in the last case one can avoid the introduction of the external source for the auxiliary scalar field. Introducing an extra constraint on the background metric, one can derive the renormalization group beta functions and explore the asymptotics of the effective charges [24.

Acknowledgments The authors are grateful to M. Asorey, I.L. Buchbinder, T. Morozumi, T. Muta and S.Odintsov for the stimulating discussions. ILS especially appreciate the contribution of B. Ovrut who paid his attention to the link between the model (11) and special version of higher derivative gravity (11). ILS is also grateful to T. Morozumi, T. Muta and to whole Department of Particle Physics for warm hospitality during his stay in Hiroshima University, and the Department of Theoretical Physics at the University of Zaragoza for warm hospitality at present time. The work of ILS has been supported in part by the RFFR (Russia), project no. 94-02-03234, and by ISF (Soros Foundation), grant RI1000. 


\section{References}

[1] T. Damour and G. Esposito-Farése, Class. Quantum Grav. 9, 2093 (1992); T. Damour and A. M. Polykov, gr-qc/9411069; To appear in Gen.Rel.Grav. (1994).

[2] M.B. Green, J.H. Schwarz and E. Witten, Superstring Theory (Cambridge University Press, Cambridge, 1987).

[3] J.D. Barrow, Nucl.Phys., B296, (1988) 697; J.D. Barrow, S. Cotsakis, Phys.Lett. 214B, (1988) 515; A.B. Burd, J.D. Barrow, Nucl.Phys., B308, (1988) 929.

[4] K. Maeda, Phys.Rev. D37, (1988) 858; J.D. Barrow and K. Maeda, Nucl.Phys. B341, (1990) 294; J.D. Barrow, Phys.Rev. D47, (1993) 5329; Phys.Rev. D48, (1993) 3592.

[5] E.J. Weinberg, Phys.Rev. D40, (1989) 3950.

[6] G.L. Cardoso and B.A. Ovrut, Natural Supergravity Inflation CERN-TH.6685/92, UPR-0526T (1992); B.A. Ovrut, Talk given on International Conference on Gravity and Field Theory, Tomsk, August 1994.

[7] T. Damour and K. Nordtvedt, Phys. Rev. D48, 3436 (1993).

[8] B. Whitt, Phys.Lett. 145B, (1984) 176.

[9] G. Magnano and L.M. Sokolowski, Phys. Rev. 50D, 5039 (1994).

[10] A.A. Starobinskii, Phys.Lett. 91B, (1980) 99; L.A. Kofman, A.D. Linde and A.A. Starobinskii, Phys.Lett. 157B, (1985) 361.

[11] Barrow J.D. and Ottewill A.C., J.Phys. A16, (1983) 2757; Barrow J.D., Phys.Lett., 183B, (1987) 285.

[12] M.B. Mijic, M.M. Morris and W.-M. Suen, Phys.Rev. D34, (1986) 2934.

[13] A.A. Starobinskii and H.J. Schmidt, Class.Quant.Grav. 4, (1987) 695.

[14] K.S. Stelle, Phys.Rev. 16D, 953 (1977).

[15] B.L. Voronov and I.V. Tyutin, Sov. J. Nucl. Phys. 23, 664 (1976).

[16] E.S. Fradkin and A.A. Tseytlin, Nucl. Phys. 201B, 469 (1982).

[17] I.G. Avramidi, Yad. Fiz. (Sov. J. Nucl. Phys.) 44, 255 (1986).

[18] I.L. Buchbinder and I.L. Shapiro, Yad. Fiz. (Sov. J. Nucl. Phys.) 44, 1033 (1986); I.L. Buchbinder, O.K. Kalashnikov, I.L. Shapiro, V.B. Vologodsky and Yu.Yu. Wolfengaut, Phys. Lett. B216, 127 (1989); I.L. Shapiro, Class. Quant. Grav. 6, 1197 (1989).

[19] I.L. Shapiro, Yad. Fiz. (Sov. J. Nucl. Phys.) (1994)

[20] S. Christensen and M. Duff., Nucl. Phys. 170B (1980) 480.

[21] B.S. DeWitt, Dynamical Theory of Groups and Fields. (Gordon and Breach, NY, 1965). 
[22] I.L. Buchbinder, S.D. Odintsov and I.L. Shapiro, Effective Action in Quantum Gravity (IOP, Bristol, 1992).

[23] G. t'Hooft and M. Veltman, Ann. Inst. H. Poincare. A20, 69 (1974).

[24] Paper in preparation.

[25] E. Elizalde, A.G. Jacksenaev, S.D. Odintsov and I.L. Shapiro, Phys. Lett. B328, 297 (1994); Preprint HUPD - 9413, Hiroshima University, 1994; E. Elizalde, S.D. Odintsov and I.L. Shapiro, Class. Quant. Grav. 11, 1607 (1994).

[26] I. Antoniadis and E. Mottola, Phys. Rev. 45D, 2013 (1992)

[27] S.D. Odintsov and I.L. Shapiro, Class. Quant. Grav. 8 L57 (1991).

[28] I.L. Buchbinder and S.D. Odintsov, Class. Quant. Grav. 2, 721 (1985).

[29] S.D. Odintsov and I.L. Shapiro, Class. Quant. Grav. 9873 (1992).

[30] I.L. Shapiro and A.G. Jacksenaev, Phys.Lett. 324B, 284 (1994).

[31] T. Aida, Y. Kitazawa, H. Kawai and M. Ninomiya, Nucl. Phys. B427 (1994) 158.

[32] T. Goldman, J. Pérez-Mercader, F. Cooper, M. Martin-Nieto, Phys. Lett. 281B (1992) 219.

[33] I.L. Shapiro, Phys.Lett. 329B, 181 (1994).

[34] S.Deser and P. van Nieuwenhuisen, Phys. Rev. 10D, 401 (1974); 10D, 411 (1974).

[35] G.A. Vilkovisky, Nucl.Phys. 234B 125 (1984).

[36] Voronov B.L., Lavrov P.M., Tyutin I.V., Sov.J.Nucl.Phys. 36498 (1992).

[37] A.O.Barvinski, A.Kamenschik, B.Karmazin, Phys.Pev. D, 48, 3677 (1993). 